\title{
Riemann-Liouville fractional integro-differential equations with fractional nonlocal integral boundary conditions
}

\author{
Bashir Ahmad ${ }^{1 *}$ and Juan J Nieto ${ }^{1,2}$
}

* Correspondence: bashir_qau@yahoo.com

'Department of Mathematics, Faculty of Science, King Abdulaziz University P.O. Box 80203, Jeddah 21589, Saudi Arabia

Full list of author information is available at the end of the article

\section{Abstract}

This article investigates a boundary value problem of Riemann-Liouville fractional integro-differential equations with fractional nonlocal integral boundary conditions. Some new existence results are obtained by applying standard fixed point theorems. 2010 Mathematics Subject Classification: 26A33; 34A34; 34B15.

Keywords: Riemann-Liouville calculus, fractional integro-differential equations, fractional boundary conditions, fixed point theorems

\section{Introduction}

In this article, we study the existence and uniqueness of solutions for the following nonlinear fractional integro-differential equation:

$$
D^{\alpha} u(t)=f(t, u(t),(\phi u)(t),(\psi u)(t)), \quad t \in[0, T], \quad \alpha \in(1,2],
$$

subject to the boundary conditions of fractional order given by

$$
\begin{aligned}
& D^{\alpha-2} u\left(0^{+}\right)=0, \\
& D^{\alpha-1} u\left(0^{+}\right)=v I^{\alpha-1} u(\eta), 0<\eta<T, \quad v \text { is a constant, }
\end{aligned}
$$

where $D^{\alpha}$ denotes the Riemann-Liouville fractional derivative of order $\alpha, f:[0, T] \times \mathbb{R}$ $\times \mathbb{R} \times \mathbb{R} \rightarrow \mathbb{R}$ is continuous, and

$$
(\phi x)(t)=\int_{0}^{t} \gamma(t, s) x(s) \mathrm{d} s, \quad(\psi x)(t)=\int_{0}^{t} \delta(t, s) x(s) \mathrm{d} s,
$$

with $\gamma$ and $\delta$ being continuous functions on $[0, T] \times[0, T]$.

Boundary value problems for nonlinear fractional differential equations have recently been investigated by several researchers. As a matter of fact, fractional derivatives provide an excellent tool for the description of memory and hereditary properties of various materials and processes (see [1]) and make the fractional-order models more realistic and practical than the classical integer-order models. Fractional differential equations arise in many engineering and scientific disciplines, such as physics, chemistry, biology, economics, control theory, signal and image processing, biophysics, blood

(c) 2011 Ahmad and Nieto; licensee Springer. This is an Open Access article distributed under the terms of the Creative Commons Attribution License (http://creativecommons.org/licenses/by/2.0), which permits unrestricted use, distribution, and reproduction in any medium, provided the original work is properly cited. 
flow phenomena, aerodynamics, fitting of experimental data, etc. (see [1,2]). For some recent development on the topic, (see [3-19] and references therein).

\section{Preliminaries}

Let us recall some basic definitions (see [20,21]).

Definition 2.1 The Riemann-Liouville fractional integral of order $\alpha>0$ for a continuous function $u:(0, \infty) \rightarrow \mathbb{R}$ is defined as

$$
I^{\alpha} u(t)=\frac{1}{\Gamma(\alpha)} \int_{0}^{t}(t-s)^{\alpha-1} u(s) \mathrm{d} s,
$$

provided the integral exists.

Definition 2.2 For a continuous function $u:(0, \infty) \rightarrow \mathbb{R}$, the Riemann-Liouville derivative of fractional order $\alpha>0, n=[\alpha]+1([\alpha]$ denotes the integer part of the real number $\alpha$ ) is defined as

$$
D^{\alpha} u(t)=\frac{1}{\Gamma(n-\alpha)}\left(\frac{1}{\mathrm{~d} t}\right)^{n} \int_{0}^{t}(t-s)^{n-\alpha-1} u(s) \mathrm{d} s=\left(\frac{\mathrm{d}}{\mathrm{d} t}\right)^{n} I^{n-\alpha} u(t)
$$

provided it exists.

For $\alpha<0$, we use the convention that $D^{\alpha} u=I^{\alpha} u$. Also for $\beta \in[0, \alpha)$, it is valid that $D^{\beta} I^{a} u=I^{\alpha-\beta} u$.

Note that for $\lambda>-1, \lambda \neq \alpha-1, \alpha-2, \ldots, \alpha-n$, we have

$$
D^{\alpha} t^{\lambda}=\frac{\Gamma(\lambda+1)}{\Gamma(\lambda-\alpha+1)} t^{\lambda-\alpha}
$$

and

$$
D^{\alpha} t^{\alpha-i}=0, \quad i=1,2, \ldots, n .
$$

In particular, for the constant function $u(t)=1$, we obtain

$$
D^{\alpha} 1=\frac{1}{\Gamma(1-\alpha)} t^{-\alpha}, \quad \alpha \notin \mathbb{N} .
$$

For $\alpha \in \mathbb{N}$, we get, of course, $D^{\alpha} 1=0$ because of the poles of the gamma function at the points $0,-1,-2, \ldots$.

For $\alpha>0$, the general solution of the homogeneous equation

$$
D^{\alpha} u(t)=0
$$

in $C(0, T) \cap L(0, T)$ is

$$
u(t)=c_{0} t^{\alpha-n}+c_{1} t^{\alpha-n-1}+\cdots+c_{n-2} t^{\alpha-2}+c_{n-1} t^{\alpha-1},
$$

where $c_{i}, i=1,2, \ldots, n-1$, are arbitrary real constants.

We always have $D^{\alpha} I^{\alpha} u=u$, and

$$
I^{\alpha} D^{\alpha} u(t)=u(t)+c_{0} t^{\alpha-n}+c_{1} t^{\alpha-n-1}+\cdots+c_{n-2} t^{\alpha-2}+c_{n-1} t^{\alpha-1} .
$$

To define the solution for the nonlinear problem (1.1) and (1.2)-(1.3), we consider the following linear equation 


$$
D^{\alpha} u(t)=\sigma(t), \alpha \in(1,2], \quad t \in[0, T], T>0,
$$

where $\sigma \in C[0, T]$.

We define

$$
A=v \int_{0}^{\eta} \frac{s^{\alpha-1}(\eta-s)^{\alpha-2}}{\Gamma(\alpha-1)} \mathrm{d} s=\frac{\nu \Gamma(\alpha) \eta^{2 \alpha-2}}{\Gamma(2 \alpha-1)}
$$

such that $A \neq \Gamma(\alpha)$.

The general solution of (2.1) is given by

$$
u(t)=c_{1} t^{\alpha-1}+c_{0} t^{\alpha-2}+I^{\alpha} \sigma(t),
$$

with $I^{\alpha}$ the usual Riemann-Liouville fractional integral of order $\alpha$.

From (2.3), we have

$$
\begin{aligned}
& D^{\alpha-1} u(t)=c_{1} \Gamma(\alpha)+I^{1} \sigma(t), \\
& D^{\alpha-2} u(t)=c_{1} \Gamma(\alpha) t+c_{0} \Gamma(\alpha-1)+I^{2} \sigma(t) .
\end{aligned}
$$

Using the conditions (1.2) and (1.3) in (2.4) and (2.5), we find that $c_{0}=0$ and

$$
c_{1}=\frac{v}{[\Gamma(\alpha)-A]} \int_{0}^{\eta} \frac{(\eta-s)^{\alpha-2}}{\Gamma(\alpha-1)}\left(\int_{0}^{s} \frac{(s-x)^{\alpha-1}}{\Gamma(\alpha)} \sigma(x) \mathrm{d} x\right) \mathrm{d} s,
$$

where $A$ is defined by (2.2).

Substituting the values of $c_{0}$ and $c_{1}$ in (2.3), the unique solution of (2.1) subject to the boundary conditions (1.2)-(1.3) is given by

$$
\begin{aligned}
u(t)= & \int_{0}^{t} \frac{(t-s)^{\alpha-1}}{\Gamma(\alpha)} \sigma(s) \mathrm{d} s \\
& +\frac{v t^{\alpha-1}}{[\Gamma(\alpha)-A]} \int_{0}^{\eta} \frac{(\eta-s)^{\alpha-2}}{\Gamma(\alpha-1)}\left(\int_{0}^{s} \frac{(s-x)^{\alpha-1}}{\Gamma(\alpha)} \sigma(x) \mathrm{d} x\right) \mathrm{d} s \\
= & \int_{0}^{t} \frac{(t-s)^{\alpha-1}}{\Gamma(\alpha)} \sigma(s) \mathrm{d} s+\frac{v t^{\alpha-1}}{[\Gamma(\alpha)-A]} I^{2 \alpha-1} \sigma(\eta) .
\end{aligned}
$$

\section{Main results}

Let $\mathcal{C}=C([0, T], \mathbb{R})$ denotes the Banach space of all continuous functions from $[0, T]$ $\rightarrow \mathbb{R}$ endowed with the norm defined by $\|u\|=\sup \{|u(t)|, t \in[0, T]\}$.

If $u$ is a solution of (1.1) and (1.2)-(1.3), then

$$
\begin{aligned}
u(t)= & \int_{0}^{t} \frac{(t-s)^{\alpha-1}}{\Gamma(\alpha)} f(s, u(s),(\phi u)(s),(\psi u)(s)) \mathrm{d} s \\
& +v_{1} t^{\alpha-1} \int_{0}^{\eta} \frac{(\eta-s)^{2 \alpha-2}}{\Gamma(2 \alpha-1)} f(s, u(s),(\phi u)(s),(\psi u)(s)) \mathrm{d} s,
\end{aligned}
$$


where

$$
v_{1}=\frac{v}{[\Gamma(\alpha)-A]} .
$$

Define an operator $\mathcal{P}: \mathcal{C} \rightarrow \mathcal{C}$ as

$$
\begin{aligned}
(\mathcal{P} u)(t)= & \int_{0}^{t} \frac{(t-s)^{\alpha-1}}{\Gamma(\alpha)} f(s, u(s),(\phi u)(s),(\psi u)(s)) \mathrm{d} s \\
& +v_{1} t^{\alpha-1} \int_{0}^{\eta} \frac{(\eta-s)^{2 \alpha-2}}{\Gamma(2 \alpha-1)} f(s, u(s),(\phi u)(s),(\psi u)(s)) \mathrm{d} s, \quad t \in[0, T] .
\end{aligned}
$$

Observe that the problem (1.1) and (1.2)-(1.3) has solutions if and only if the operator equation $\mathcal{P} u=u$ has fixed points.

Lemma 3.1 The operator Pis compact.

Proof

(i) Let $\mathbf{B}$ be a bounded set in $C[0, T]$. Then, there exists a constant $M$ such that $f$ $(t, u(t),(\phi u)(t),(\psi u)(t)) \mid \leq M, \forall \mathbf{u} \in \mathbf{B}, t \in[0, T]$. Thus

$$
\begin{aligned}
|(\mathcal{P} u)(t)| & \leq M \int_{0}^{t} \frac{(t-s)^{\alpha-1}}{\Gamma(\alpha)} \mathrm{d} s+M\left|v_{1}\right| t^{\alpha-1} \int_{0}^{\eta} \frac{(\eta-s)^{2 \alpha-2}}{\Gamma(2 \alpha-1)} \mathrm{d} s \\
& \leq M T^{\alpha-1}\left(\frac{T}{\Gamma(\alpha+1)}+\frac{\left|v_{1}\right| \eta^{2 \alpha-1}}{\Gamma(2 \alpha)}\right)
\end{aligned}
$$

which implies that

$$
\|(\mathcal{P} u)\| \leq M T^{\alpha-1}\left(\frac{T}{\Gamma(\alpha+1)}+\frac{\left|v_{1}\right| \eta^{2 \alpha-1}}{\Gamma(2 \alpha)}\right)<\infty .
$$

Hence, $\mathcal{P}(\mathbf{B})$ is uniformly bounded.

(ii) For any $t_{1}, t_{2} \in[0, T], u \in \mathbf{B}$, we have

$$
\begin{aligned}
& \left|(\mathcal{P} u)\left(t_{1}\right)-(\mathcal{P} u)\left(t_{2}\right)\right| \\
& =\mid \int_{0}^{t_{1}} \frac{\left(t_{1}-s\right)^{\alpha-1}}{\Gamma(\alpha)} f(s, u(s),(\phi u)(s),(\psi u)(s)) \mathrm{d} s \\
& \quad-\int_{0}^{t_{2}} \frac{\left(t_{2}-s\right)^{\alpha-1}}{\Gamma(\alpha)} f(s, u(s),(\phi u)(s),(\psi u)(s)) \mathrm{d} s \\
& \quad+v_{1}\left(t_{1}^{\alpha-1}-t_{2}^{\alpha-1}\right) \int_{0}^{\eta} \frac{(\eta-s)^{2 \alpha-s}}{\Gamma(2 \alpha-1)} f(s, u(s),(\phi u)(s),(\psi u)(s)) \mathrm{d} s \mid \\
& \leq M\left(\left|\frac{1}{\Gamma(\alpha)} \int_{0}^{t_{1}}\left[\left(t_{1}-s\right)^{\alpha-1}-\left(t_{2}-s\right)^{\alpha-1}\right] \mathrm{d} s-\frac{1}{\Gamma(\alpha)} \int_{t_{1}}^{t_{2}}\left(t_{2}-s\right)^{\alpha-1} \mathrm{~d} s\right|\right. \\
& \left.\quad+\left|v_{1}\left(t_{1}^{\alpha-1}-t_{2}^{\alpha-1}\right) \int_{0}^{\eta} \frac{(\eta-s)^{2 \alpha-s}}{\Gamma(2 \alpha-1)} \mathrm{d} s\right|\right) \rightarrow 0 \text { as } t_{1} \rightarrow t_{2} .
\end{aligned}
$$


Thus, $\mathcal{P}(\mathbf{B})$ is equicontinuous. Consequently, the operator $\mathcal{P}$ is compact. This completes the proof.

We need the following known fixed point theorem to prove the existence of solutions for the problem at hand.

Theorem 3.1 ([22]) Let $E$ be a Banach space. Assume that T: $E \rightarrow E$ be a completely continuous operator and the set $V=\{x \in E \mid x=\mu T x, 0<\mu<1\}$ be bounded.

Then, $T$ has a fixed point in $E$.

Theorem 3.2 Assume that there exists a constant $M>0$ such that

$$
|f(t, u(t),(\phi u)(t),(\psi u)(t))| \leq M, \quad \forall t \in[0, T], \quad u \in \mathbb{R} .
$$

Then, the problem (1.1) and (1.2)-(1.3) has at least one solution on [0,T].

Proof We consider the set

$$
V=\{u \in \mathbb{R} \mid u=\mu \mathcal{P} u, \quad 0<\mu<1\},
$$

and show that the set $V$ is bounded. Let $u \in V$, then $u=\mu \mathcal{P} u, 0<\mu<1$. For any $t \in$ $[0, T]$, we have

$$
\begin{aligned}
|u(t)| \leq & \mu\left[\int_{0}^{t} \frac{(t-s)^{\alpha-1}}{\Gamma(\alpha)}|f(s, u(s),(\phi u)(s),(\psi u)(s))| \mathrm{d} s\right. \\
& \left.+\left|v_{1}\right| t^{\alpha-1} \int_{0}^{\eta} \frac{(\eta-s)^{2 \alpha-2}}{\Gamma(2 \alpha-1)}|f(s, u(s),(\phi u)(s),(\psi u)(s))| \mathrm{d} s\right] .
\end{aligned}
$$

As in part (i) of Lemma 3.1, we have

$$
\|(\mathcal{P} u)\| \leq M T^{\alpha-1}\left(\frac{T}{\Gamma(\alpha+1)}+\frac{\left|v_{1}\right| \eta^{2 \alpha-1}}{\Gamma(2 \alpha)}\right)<\infty .
$$

This implies that the set $V$ is bounded independently of $\mu \in(0,1)$. Using Lemma 3.1 and Theorem 3.1, we obtain that the operator $\mathcal{P}$ has at least a fixed point, which implies that the problem (1.1) and (1.2)-(1.3) has at least one solution. This completes the proof.

Theorem 3.3 Assume that

$\left(\mathrm{A}_{1}\right)$ there exist positive functions $L_{1}(t), L_{2}(t), L_{3}(t)$ such that

$$
\begin{aligned}
& |f(t, u(t),(\phi u)(t),(\psi u)(t))-f(t, v(t),(\phi v)(t),(\psi v)(t))| \\
& \leq L_{1}(t)|u-v|+L_{2}(t)|\phi u-\phi v|+L_{3}(t)|\psi u-\psi v|, \quad \forall t \in[0,1], \quad u, v \in \mathbb{R} .
\end{aligned}
$$

$\left(\mathrm{A}_{2}\right) \Lambda=\left(\xi_{1}+\left|v_{1}\right| T^{\alpha-1} \xi_{2}\right)\left(1+\gamma_{0}+\delta_{0}\right)<1$, where

$$
\begin{aligned}
& \gamma_{0}=\sup _{t \in[0,1]}\left|\int_{0}^{t} \gamma(t, s) \mathrm{d} s\right|, \quad \delta_{0}=\sup _{t \in[0,1]}\left|\int_{0}^{t} \delta(t, s) \mathrm{d} s\right|, \\
& \xi_{1}=\sup _{t \in[0, T]}\left\{\left|I^{q} L_{1}(t)\right|, \quad\left|I^{q} L_{2}(t)\right|, \quad\left|I^{q} L_{3}(t)\right|\right\}, \\
& \xi_{2}=\max \left\{\left|I^{2 \alpha-1} L_{1}(\eta)\right|, \quad\left|I^{2 \alpha-1} L_{2}(\eta)\right|, \quad\left|I^{2 \alpha-1} L_{3}(\eta)\right|\right\},
\end{aligned}
$$

Then the problem (1.1) and (1.2)-(1.3) has a unique solution on $C[0, T]$.

Proof Let us set $\sup _{t \in[0, T]}|f(t, 0,0,0)|=M$, and choose

$$
r \geq \frac{\varepsilon M}{1-\Lambda}
$$


Then we show that $\mathcal{P} B_{r} \subset B_{r}$, where $B_{r}=\{x \in \mathcal{C}:\|u\| \leq r\}$. For $x L B_{r}$, we have

$$
\begin{aligned}
& \|(\mathcal{P} u)(t)\|=\sup _{t \in[0, T]} \mid \int_{0}^{t} \frac{(t-s)^{\alpha-1}}{\Gamma(\alpha)} f(s, u(s),(\phi u)(s),(\psi u)(s)) \mathrm{d} s \\
& +v_{1} t^{\alpha-1} \int_{0}^{\eta} \frac{(\eta-s)^{2 \alpha-2}}{\Gamma(2 \alpha-1)} f(s, u(s),(\phi u)(s),(\psi u)(s)) \mathrm{d} s \mid \\
& \leq \sup _{t \in[0, T]}\left(\int_{0}^{t} \frac{(t-s)^{\alpha-1}}{\Gamma(\alpha)}(|f(s, x(s),(\phi x)(s),(\psi x)(s))-f(s, 0,0,0)|\right. \\
& +|f(s, 0,0,0)|) \mathrm{d} s \\
& +\left|v_{1}\right| t^{\alpha-1} \int_{0}^{\eta} \frac{(\eta-s)^{2 \alpha-s}}{\Gamma(2 \alpha-1)}(\mid f(s, x(s),(\phi x)(s),(\psi x)(s))-f(s, 0,0,0) \\
& +|f(s, 0,0,0)|) \mathrm{d} s) \\
& \leq \sup _{t \in[0, T]}\left(\int_{0}^{t} \frac{(t-s)^{\alpha-1}}{\Gamma(\alpha)}\left(L_{1}(s)|x(s)|+L_{2}(s)|(\phi x)(s)|+L_{3}(s)|(\psi x)(s)|+M\right) \mathrm{d} s\right. \\
& \left.+\left|\nu_{1}\right| t^{\alpha-1} \int_{0}^{\eta} \frac{(\eta-s)^{2 \alpha-2}}{\Gamma(2 \alpha-1)}\left(L_{1}(s)|x(s)|+L_{2}(s)|(\phi x)(s)|+L_{3}(s)|(\psi x)(s)|+M\right) \mathrm{d} s\right) \\
& \leq \sup _{t \in[0, T]}\left(\int _ { 0 } ^ { t } \frac { ( t - s ) ^ { \alpha - 1 } } { \Gamma ( \alpha ) } \left(L_{1}(s)|x(s)|+\gamma_{0} L_{2}(s)|x(s)|\right.\right. \\
& \left.+\delta_{0} L_{3}(s)|x(s)|+M\right) \mathrm{d} s \\
& +\left|v_{1}\right| t^{\alpha-1} \int_{0}^{\eta} \frac{(\eta-s)^{2 \alpha-2}}{\Gamma(2 \alpha-1)}\left(L_{1}(s)|x(s)|+\gamma_{0} L_{2}(s)|x(s)|\right. \\
& \left.\left.+\delta_{0} L_{3}(s)|x(s)|+M\right) \mathrm{~d} s\right) \\
& \leq \sup _{t \in[0, T]}\left(\left(I^{\alpha} L_{1}(t)+\gamma_{0} I^{\alpha} L_{2}(t)+\delta_{0} I^{\alpha} L_{3}(t)\right) r+\frac{M t^{q}}{\Gamma(q+1)}\right. \\
& \left.+\left|\nu_{1}\right| t^{\alpha-1}\left(I^{(2 \alpha-1)} L_{1}(\eta)+\gamma_{0} I^{(2 \alpha-1)} L_{2}(\eta)+\delta_{0} I^{(2 \alpha-1)} L_{3}(\eta)\right) r+\frac{M \eta^{2 \alpha-1}}{\Gamma(2 \alpha)}\right) \\
& \leq\left(\xi_{1}+\left|v_{1}\right| T^{\alpha-1} \xi_{2}\right)\left(1+\gamma_{0}+\delta_{0}\right) r+M T^{\alpha-1}\left(\frac{T}{\Gamma(\alpha+1)}+\frac{\left|v_{1}\right| \eta^{\alpha-1}}{\Gamma(2 \alpha)}\right) \\
& =\Lambda r+M \varepsilon \leq r
\end{aligned}
$$

In view of $\left(A_{1}\right)$, for every $t \in[0, T]$, we have

$$
\begin{aligned}
& |(\mathcal{P} u)(t)-(\mathcal{P} v)(t)| \\
& \leq \sup _{t \in[0, T]}\left(\int_{0}^{t} \frac{(t-s)^{\alpha-1}}{\Gamma(\alpha)} \mid f(s, u(s),(\phi u)(s),(\psi u)(s)-f(s, v(s),(\phi v)(s),(\psi v)(s)) \mid \mathrm{d} s\right. \\
& \quad+\left|v_{1}\right| t^{\alpha-1} \int_{0}^{\eta} \frac{(\eta-s)^{2 \alpha-2}}{\Gamma(2 \alpha-1)} \mid f(s, u(s),(\phi u)(s),(\psi u)(s)-f(s, v(s),(\phi v)(s),(\psi v)(s)) \mid \mathrm{d} s) \\
& \leq \quad \sup _{t \in[0, T]}\left(\int_{0}^{t} \frac{(t-s)^{\alpha-1}}{\Gamma(\alpha)}\left(L_{1}(s)|u-v|+L_{2}(s)|\phi v|+L_{3}(s)|\psi u-\psi v|\right) \mathrm{d} s\right. \\
& \left.\quad+\left|v_{1}\right| t^{\alpha-1} \int_{0}^{\eta} \frac{(\eta-s)^{2 \alpha-2}}{\Gamma(2 \alpha-1)} \mid\left(L_{1}(s)|u-v|+L_{2}(s)|\phi u-\phi v|+L_{3}(s)|\psi u-\psi v|\right) \| \mathrm{d} s\right) \\
& \leq \sup _{t \in[0, T]}\left(\left(I^{\alpha} L_{1}(t)+\gamma_{0} I^{\alpha} L_{2}(t)+\delta_{0} I^{\alpha} L_{3}(t)\right)\|u-v\|\right. \\
& \quad+\left|v_{1}\right| T^{\alpha-1}\left(I^{(2 \alpha-1)} L_{1}(\eta)+\gamma_{0} I^{(2 \alpha-1)} L_{2}(\eta)+\delta_{0} I^{(2 \alpha-1)} L_{3}(\eta)\right)\|u-v\| \\
& \leq \\
& \quad\left(\xi_{1}+\left|v_{1}\right| T^{\alpha-1} \xi_{2}\right)\left(1+\gamma_{0}+\delta_{0}\right)\|u-v\|=\Lambda\|u-v\|
\end{aligned}
$$


By assumption $\left(A_{2}\right), \Lambda<1$, therefore, the operator $\mathcal{P}$ is a contraction. Hence, by Banach fixed point theorem, we deduce that $\mathcal{P}$ has a unique fixed point which in fact is a unique solution of problem (1.1) and (1.2)-(1.3). This completes the proof.

Theorem 3.4 (Krasnoselskii's fixed point theorem [22]). Let $\mathcal{M} b e$ a closed convex and nonempty subset of a Banach space X. Let A, B be the operators such that (i) $x, y \in \mathcal{M}$ whenever $x, y \in \mathcal{M}$; (ii) $A$ is compact and continuous; (iii) $B$ is a contraction mapping. Then, there exists $z \in \mathcal{M}$ such that $z=A z+B z$.

Theorem 3.5 Assume that $f:[0, T] \times \mathbb{R} \times \mathbb{R} \times \mathbb{R} \rightarrow \mathbb{R}$ is a continuous function and the following assumptions hold:

$\left(\mathbf{H}_{1}\right)$

$$
\begin{aligned}
& |f(t, u(t),(\phi u)(t),(\psi u)(t))-f(t, v(t),(\phi v)(t),(\psi v)(t))| \\
& \leq L_{1}(t)|u-v|+L_{2}(t)|\phi u-\phi v|+L_{3}(t)|\psi u-\psi v|, \quad \forall t \in[0, T], u, v \in \mathbb{R} .
\end{aligned}
$$

$\left(\mathbf{H}_{2}\right)|f(t, u)| \leq \mu(t), \forall(t, u) \in[0, T] \times \mathbb{R}$, and $\mu \in C\left([0, T], \mathbb{R}^{+}\right)$.

If

$$
\frac{\left|v_{1}\right| T^{\alpha-1} \eta^{2 \alpha-1}}{\Gamma(2 \alpha)}<1
$$

then the boundary value problem (1.1) and (1.2)-(1.3) has at least one solution on [0, T].

Proof Letting $\sup _{t \in[0, T]}|\mu(t)|=|| \mu||$, we fix

$$
\bar{r} \geq\|\mu\| T^{\alpha-1}\left(\frac{T}{\Gamma(\alpha+1)}+\frac{\left|\nu_{1}\right| \eta^{2 \alpha-1}}{\Gamma(2 \alpha)}\right),
$$

and consider $B_{\bar{r}}=\{u \in \mathcal{C}:\|u\| \leq \bar{r}\}$. We define the operators $\mathcal{P}_{1}$ and $\mathcal{P}_{2}$ on $B_{\bar{r}}$ as

$$
\begin{aligned}
& \left(\mathcal{P}_{1} u\right)(t)=\int_{0}^{t} \frac{(t-s)^{\alpha-1}}{\Gamma(\alpha)} f(s, u(s),(\phi u)(s),(\psi u)(s)) \mathrm{d} s \\
& \left(\mathcal{P}_{2} u\right)(t)=v_{1} t^{\alpha-1} \int_{0}^{\eta} \frac{(\eta-s)^{2 \alpha-s}}{\Gamma(2 \alpha-1)} f(s, u(s),(\phi u)(s),(\psi u(s)) \mathrm{d} s) .
\end{aligned}
$$

For $u, v \in B_{\bar{r}}$, we find that

$$
\left\|\mathcal{P}_{1} u+\mathcal{P}_{2} v\right\| \leq\|\mu\| T^{\alpha-1}\left(\frac{T}{\Gamma(\alpha+1)}+\frac{\left|v_{1}\right| \eta^{2 \alpha-1}}{\Gamma(2 \alpha)}\right) \leq \bar{r} .
$$

Thus, $\mathcal{P}_{1} u+\mathcal{P}_{2} v \in B_{\bar{r}}$. It follows from the assumption $\left(H_{1}\right)$ together with (3.1) that $\mathcal{P}_{2}$ is a contraction mapping. Continuity of $f$ implies that the operator $\mathcal{P}_{1}$ is continuous.

Also, $\mathcal{P}_{1}$ is uniformly bounded on $B_{\bar{r}}$ as

$$
\left\|\mathcal{P}_{1} u\right\| \leq \frac{\|u\| T^{\alpha}}{\Gamma(\alpha+1)}
$$

Now we prove the compactness of the operator $\mathcal{P}_{1}$.

In view of $\left(H_{1}\right)$, we define $\sup _{(t, x, \phi x, \psi x) \in[0, T] \times B_{r} \times B_{r} \times B_{r}}|f(t, x, \phi x, \psi x)|=\bar{f}$, and consequently we have 


$$
\begin{aligned}
& \left|\left(\mathcal{P}_{1} u\right)\left(t_{1}\right)-\left(\mathcal{P}_{2} u\right)\left(t_{2}\right)\right| \\
= & \mid \frac{1}{\Gamma(\alpha)} \int_{0}^{t_{1}}\left[\left(t_{1}-s\right)^{\alpha-1}-\left(t_{2}-s\right)^{\alpha-1}\right](s, u(s),(\phi u)(s),(\psi u)(s)) \mathrm{d} s \\
& -\frac{1}{\Gamma(\alpha)} \int_{t_{1}}^{t_{2}}\left(t_{2}-s\right)^{\alpha-1} f(s, u(s),(\phi u)(s),(\psi u)(s)) \mathrm{d} s \mid \\
\leq & \frac{\bar{f}}{\Gamma(\alpha+1)}\left|2\left(t_{2}-t_{1}\right)^{\alpha}+t_{1}^{\alpha}-t_{2}^{\alpha}\right|,
\end{aligned}
$$

which is independent of $u$ and tends to zero as $t_{2} \rightarrow t_{1}$. So, $\mathcal{P}_{1}$ is relatively compact on $B_{\bar{r}}$. Hence, by the Arzelá-Ascoli Theorem, $\mathcal{P}_{1}$ is compact on $B_{\bar{r}}$. Thus, all the assumptions of Theorem 3.4 are satisfied. So the conclusion of Theorem 3.4 implies that the boundary value problem (1.1) and (1.2)-(1.3) has at least one solution on [0, $T]$. This completes the proof.

\section{Acknowledgements}

This study was partially supported by Deanship of Scientific Research (DSR), King Abdulaziz University, Jeddah, Saudi Arabia.

\section{Author details}

'Department of Mathematics, Faculty of Science, King Abdulaziz University P.O. Box 80203, Jeddah 21589, Saudi Arabia ${ }^{2}$ Departamento de Análisis Matemático, Facultad de Matemáticas Universidad de Santiago de Compostela, Santiago de Compostela 15782, Spain

\section{Authors' contributions}

Both authors, BA and JJN, contributed to each part of this work equally and read and approved the final version of the manuscript.

\section{Competing interests}

The authors declare that they have no competing interests.

\section{Received: 20 July 2011 Accepted: 17 October 2011 Published: 17 October 2011}

\section{References}

1. Podlubny, I: Fractional Differential Equations. Academic Press, San Diego (1999)

2. Sabatier, J, Agrawal, OP, Machado, JAT, (eds): Advances in Fractional Calculus: Theoretical Developments and Applications in Physics and Engineering. Springer, Dordrecht (2007)

3. Agarwal, RP, Andrade, B, Cuevas, C: Weighted pseudo-almost periodic solutions of a class of semilinear fractional differential equations. Nonlinear Anal Real World Appl. 11, 3532-3554 (2010). doi:10.1016/j.nonrwa.2010.01.002

4. Agarwal, RP, Lakshmikantham, V, Nieto, JJ: On the concept of solution for fractional differential equations with uncertainty. Nonlinear Anal. 72, 2859-2862 (2010). doi:10.1016/j.na.2009.11.029

5. Ahmad, B, Nieto, JJ: Existence results for nonlinear boundary value problems of fractional integrodifferential equations with integral boundary conditions. Bound Value Probl 2009, 11 (2009). (Article ID 708576)

6. Ahmad, B, Nieto, JJ: Existence results for a coupled system of nonlinear fractional differential equations with three-point boundary conditions. Comput Math Appl. 58, 1838-1843 (2009). doi:10.1016/j.camwa.2009.07.091

7. Ahmad, B, Nieto, JJ: Existence of solutions for anti-periodic boundary value problems involving fractional differential equations via Leray-Schauder degree theory. Topol Methods Nonlinear Anal. 35, 295-304 (2010)

8. Ahmad, B: Existence of solutions for irregular boundary value problems of nonlinear fractional differential equations. Appl Math Lett. 23, 390-394 (2010). doi:10.1016/j.aml.2009.11.004

9. Ahmad, B, Alsaedi, A: Existence and uniqueness of solutions for coupled systems of higher-order nonlinear fractional differential equations. Fixed Point Theory Appl 2010, 17 (2010). (Article ID 364560)

10. Ahmad, B, Nieto, JJ: Riemann-Liouville fractional differential equations with fractional boundary conditions. Fixed Point Theory. (in press)

11. Ahmad, B, Nieto, JJ: Anti-periodic fractional boundary value problems. Comput Math Appl. 62, 1150-1156 (2011). doi:10.1016/j.camwa.2011.02.034

12. Arciga-Alejandre, MP: Asymptotics for nonlinear evolution equation with module-fractional derivative on a half-line. Bound Value Probl 2011, 29 (2011). (Article ID 946143). doi:10.1186/1687-2770-2011-29

13. Bai, Z: On positive solutions of a nonlocal fractional boundary value problem. Nonlinear Anal. 72, 916-924 (2010). doi:10.1016/j.na.2009.07.033

14. Belmekki, M, Nieto, JJ, Rodríguez-López, R: Existence of periodic solution for a nonlinear fractional differential equation. Bound Value Probl 2009, 18 (2009). (Article ID 324561) 
15. De la Sen, M: About robust stability of Caputo linear fractional dynamic systems with time delays through fixed point theory. Fixed Point Theory Appl 2011, 19 (2011). (Article ID 867932). doi:10.1186/1687-1812-2011-19

16. Nieto, Jj: Maximum principles for fractional differential equations derived from Mittag-Leffler functions. Appl Math Lett. 23, 1248-1251 (2010). doi:10.1016/j.aml.2010.06.007

17. Wang, Y, Wang, F, An, Y: Existence and multiplicity of positive solutions for a nonlocal differential equation. Bound Value Probl. 2011, 5 (2011). doi:10.1186/1687-2770-2011-5

18. Wei, Z, Li, Q, Che, J: Initial value problems for fractional differential equations involving Riemann-Liouville sequential fractional derivative. J Math Anal Appl. 367, 260-272 (2010). doi:10.1016/j.jmaa.2010.01.023

19. Zhang, S: Positive solutions to singular boundary value problem for nonlinear fractional differential equation. Comput Math Appl. 59, 1300-1309 (2010)

20. Kilbas, AA, Srivastava, HM, Trujillo, JJ: Theory and Applications of Fractional Differential Equations. In North-Holland Mathematics Studies, vol. 204,Elsevier, Amsterdam (2006)

21. Samko, SG, Kilbas, AA, Marichev, Ol: Fractional Integrals and Derivatives, Theory and Applications. Gordon and Breach, Yverdon (1993)

22. Smart, DR: Fixed Point Theorems. Cambridge University Press, Cambridge (1980)

doi:10.1186/1687-2770-2011-36

Cite this article as: Ahmad and Nieto: Riemann-Liouville fractional integro-differential equations with fractional nonlocal integral boundary conditions. Boundary Value Problems 2011 2011:36.

\section{Submit your manuscript to a SpringerOpen ${ }^{\circ}$} journal and benefit from:

- Convenient online submission

- Rigorous peer review

- Immediate publication on acceptance

- Open access: articles freely available online

- High visibility within the field

- Retaining the copyright to your article

Submit your next manuscript at $\gg$ springeropen.com 